\title{
Actin Colocalization with Metaphase Chromosomes of the Second Meiosis in Ovulated Mouse Oocytes
}

\author{
Natalie Bogolyubova and Alexander Ginzburg \\ Institute of Cytology, Russian Academy of Sciences, Tikhoretsky Avenue 4, Saint Petersburg 194064, Russia \\ Correspondence should be addressed to Natalie Bogolyubova; natbo.cm@gmail.com
}

Received 13 November 2012; Accepted 18 February 2013

Academic Editor: Tetsuya Kojima

Copyright ( 2013 N. Bogolyubova and A. Ginzburg. This is an open access article distributed under the Creative Commons Attribution License, which permits unrestricted use, distribution, and reproduction in any medium, provided the original work is properly cited.

\begin{abstract}
Functional interrelation of nuclear actin with transcriptional active chromatin in the interphase nucleus was reliably established in numerous experiments, but the relationship between actin and transcriptional silent chromatin is still unclear. We examined localization area of the second meiotic division metaphase plate in ovulated mouse oocytes with the aim to study the possibility of actin-chromatin colocalization and uncovering the distribution patterns of different functional forms of actin near the metaphase chromosomes. Confocal microscopy and probes for actin that are distinguished from each other by the mechanism of actin binding (TRITC-phalloidin, fluorescent DNase-I, and antibodies against fragment of C-terminal and fragment of N-terminal domain of actin) were used for actin visualization. Despite the fact that TRITC-phalloidin could not detect F-actin in the area of metaphase plate, oocytes staining with antibody against fragment of the actin $\mathrm{N}$-terminal domain demonstrates the presence near the metaphase chromosomes of some spindle-like structure composed of actin filaments. Among all used probes for actin, only the antibody against fragment of the C-terminal domain detected accurate actin colocalization with metaphase chromosomes. We conclude that this antibody labeled noncanonical form of the nuclear actin existing in long-term association with highly condensed chromatin.
\end{abstract}

\section{Introduction}

Actin is an important participant of eukaryotic cell metabolism. Within the nucleus actin exists in several functional forms-monomeric (G-actin), actin oligomers, and short filaments [1]. The presence of canonical filamentous actin (F-actin) in the nucleus under normal conditions has not been authentically proved.

Results of numerous studies carried out with the use of various somatic cell types have demonstrated involvement of nuclear actin to such basic nuclear processes as mRNA processing, nuclear export, and intranuclear transport $[2,3]$. By the present time convincing evidence of actin association with functionally active chromatin was obtained: actin was identified as a part of all three RNA polymerases [4] and of chromatin remodeling complexes [5]. But at the same time interrelations between actin and transcriptional silent highly condensed chromatin (or chromosomes) are still unclear.

The functional role of actin in nuclear metabolism during gametogenesis and in initial stages of embryo development today is poorly understood. Historically the main part of data on cellular biology of nuclear actin has been received in experiments designed with the use of cultured somatic cells or in situ models, and much less works were performed on early animal embryos or gametes [6-8].

In this study which is performed on ovulated mouse oocytes, functional forms of actin which are located in the area of meiosis II metaphase plate were identified at light microscopic level and patterns of their distribution were examined. A special attention has been given to actinchromatin colocalization.

\section{Materials and Methods}

The experiments were carried out on BALB/c mice taken from the animal nursery (Rappolovo, Russia). Females were received at 6 weeks of age and were acclimated for 2 weeks. Mice were housed at a density of 5 per cage in wellventilated polycarbonate cages on wood chips, in a constant 
temperature facility and have been provided with filtered water and laboratory chow. All experiments performed in this study were conducted in accordance with the National Rules of the Laboratory Procedure with the Use of Experimental Animals confirmed by the Ministry of Public Health, Order 755.

Females were induced to ovulate by sequential single injections of serum (Folligon, Intervet, Holland) and chorionic (Chorulon, Intervet, Holland) gonadotropins, 5-10 units per animal, with $44-48 \mathrm{~h}$ interval. The oocytes age was calculated from the time of chorionic gonadotropin injection. In $18 \mathrm{~h}$ after chorionic gonadotropin injection cumulus-oocyte complexes were isolated from oviducts and were placed into F10 medium with $25 \mathrm{mM}$ HEPES (Sigma, USA). The oocytes without visible morphological defects were selected and used in experiments.

For depolymerization of actin filaments, the oocytes after explantation were placed in $10 \mu \mathrm{M}$ latrunculin B (Sigma, USA) in F12 medium and then were incubated during 1 hour under $5 \% \mathrm{CO}_{2}$ environment at $37^{\circ} \mathrm{C}$.

For indirect immunofluorescent analysis, A2066 rabbit polyclonal antibody to the fragment of actin C-terminal domain (Sigma, USA), dilution 1:100, and A2103 rabbit polyclonal antibody to the fragment of actin $\mathrm{N}$-terminal domain (Sigma, USA), dilution 1:200, were applied. FITCconjugated goat antibodies to rabbit immunoglobulins (Sigma, USA) were used as secondary antibody. Alexa Fluor 488-DNase I (Molecular Probes, USA) which specifically binds with monomeric actin was applied $(9 \mu \mathrm{g} / \mathrm{mL})$ to determine G-actin. F-actin was identified with the use of $10 \mu \mathrm{g} / \mathrm{mL}$ solution of TRITC-phalloidin (Sigma, USA).

To identify actin by indirect immunofluorescence, oocytes were fixed in $4 \%$ paraformaldehyde on PBS for 40-60 min at room temperature and then were washed three times in PBS. Prior to fixation, the attached cumulus cells were removed gently with short incubation in F10 medium containing $0.5 \%$ hyaluronidase (Sigma, USA). After that cells were permeabilized with $0.5 \%$ Triton X-100 in PBS for $10 \mathrm{~min}$, were washed three times in PBS, and were incubated in $10 \%$ fetal calf serum to block unspecific antibody binding. Then oocytes were incubated with primary antibodies at $4^{\circ} \mathrm{C}$ for not less than $12 \mathrm{~h}$. Oocytes washed in PBS and incubated with $10 \%$ fetal calf serum for $10 \mathrm{~min}$ were placed in secondary antibodies for $60 \mathrm{~min}$ at room temperature. Samples washed in PBS and stained with $1 \mu \mathrm{g} / \mathrm{mL}$ TO-PRO-3 (Molecular Probes, USA) for DNA detection were mounted on slides in VectaShield medium (Vector Laboratories, USA). Preparations were analyzed under scanning confocal microscope Leica TSC SL. Oocytes stained only with secondary antibodies served as control.

G-actin was identified by incubation of fixed and permeabilized oocytes with Alexa Fluor 488-DNAase-I conjugate for $1 \mathrm{~h}$ at room temperature. Then samples were washed three times in PBS and mounted on slides for the assay. F-actin was visualized by TRITC-phalloidin for $1 \mathrm{~h}$ at room temperature. Samples were assayed under confocal microscope Leica TSC SL.

\section{Results and Discussion}

For intracellular actin visualization four dyes with four different ways of actin binding have been chosen. As a result, oocytes labeling by TRITC-phalloidin and fluorescent DNase-I uncovers distribution only of canonical forms (Factin and G-actin, resp.), and indirect immunofluorescence with antibodies against fragments of C-terminal and $\mathrm{N}$ terminal actin domains is capable to demonstrate localization of all actin structures which contain actin molecules with opened for antibody binding epitopes at $\mathrm{C}$ or $\mathrm{N}$ ends. Subsequent comparative analysis of actin distribution patterns received after oocytes treatment by each marker allows to establish sites of localization of different functional actin forms, both canonical and uncommon.

Double staining of oocytes by G-actin marker Alexa Fluor488-DNAase-I and DNA marker TO-PRO-3 demonstrat-ed the uniform distribution of G-actin around metaphase chromosomes and the absence of actin-chromosomes colocalization (Figure 1(c)). Dispersed actin distribution near metaphase plate strongly differs from clustered distribution as a whole in cytoplasm and that makes the spindle location well appreciable (Figure 1(c)).

Staining of F-actin and chromatin by TRITC-phalloidin and TO-PRO-3 visualized in the cells typical cortical layer of microfilaments. Actin filaments were not observed around metaphase plate (Figure 1(d)), and a small number of Factin clusters were present in the cytoplasm. All metaphase chromosomes labeled by TO-PRO-3 stayed unlabeled by TRITC-phalloidin, pointing to the absence of colocalization between F-actin and chromatin (Figure 1(f)).

Antibody to the fragment of $\mathrm{N}$-terminal domain of actin has recognized cortical F-actin layer and cytoplasmic actin, but actin fluorescence has not shown any signs of colocalization with chromosomes stained by TO-PRO-3 (Figure 1(i)). At the same time, actin filaments were clearly observed on both sides of metaphase plate. They formed spindlelike structure bearing some resemblance to the microtubule spindle (Figure $1(j))$.

After oocytes labeling with antibody to the fragment of C-terminal domain, cortical layer of F-actin was not observed, and it was possible to see numerous actin clusters diffusely distributed in the cytoplasm with the bright fluorescence of antibody-reacted actin in the metaphase plate area (Figure 2(a)). Double staining of actin and DNA shows an accurate colocalization of actin with metaphase chromosomes (Figure 2(c)). The study of destructed metaphase plates in squashed oocytes discovered incompleteness of this colocalization, as fluorescence of actin was not detected in centromere regions of chromosomes (Figure 2(f)).

Latrunculin B was initially identified as an inhibitor of actin polymerization [9]. Effect of this drug is based on sequestration of monomeric actin in 1:1 molar complex. Binding latrunculin B to G-actin prevents participation of monomers in actin polymerization and as a result one can observe disruption of F-actin cytoskeleton in the cell. After $1 \mathrm{~h}$ incubation with $10 \mu \mathrm{M}$ latrunculin $\mathrm{B}$ oocytes stained by TRITC-phalloidin and antibody to $\mathrm{N}$-terminal domain 


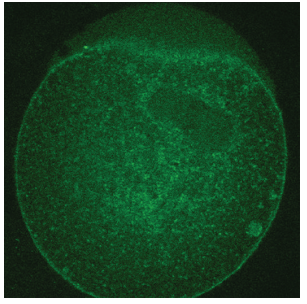

(a)

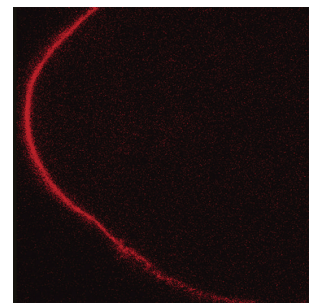

(d)

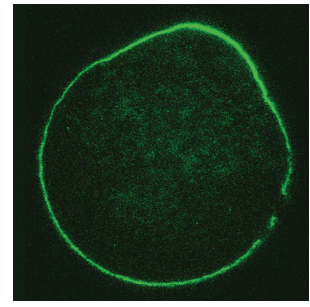

(g)

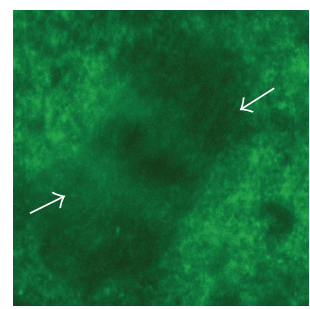

(j)

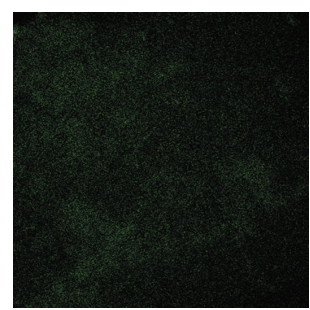

(m)

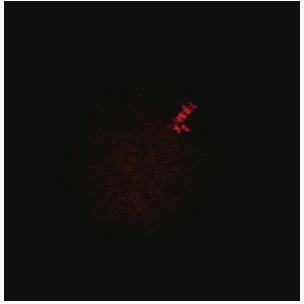

(b)

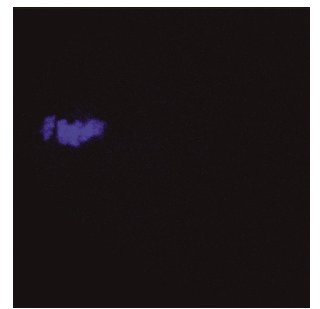

(e)

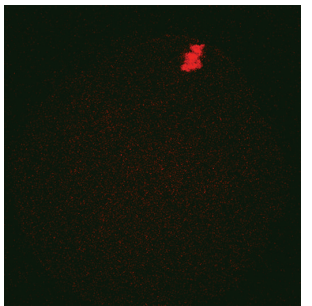

(h)

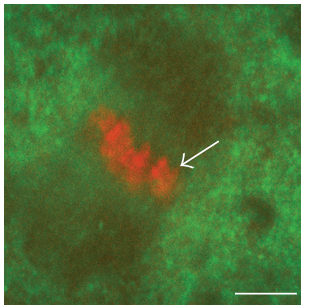

(k)

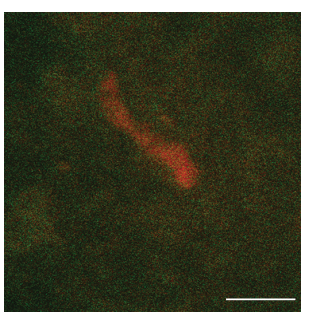

(n)

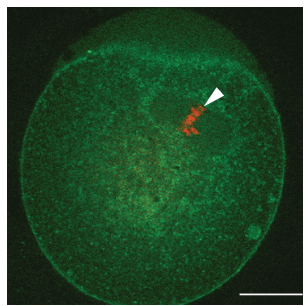

(c)

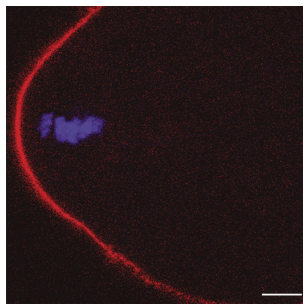

(f)

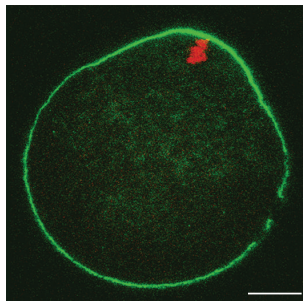

(i)

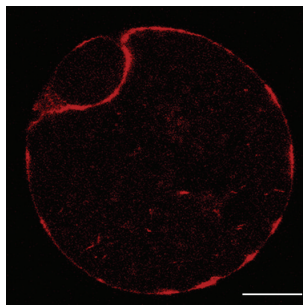

(1)

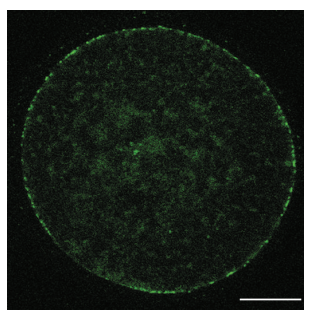

(o)

FIgURE 1: Actin distribution in the area of meiosis II metaphase plate. (a-c) Monomeric actin distribution in oocytes stained with Alexa Fluor 488-DNase-I for G-actin and TO-PRO-3 for DNA; (a) actin fluorescence after Alexa Fluor 488-DNase-I labeling; (b) DNA staining by TO-PRO-3; (c) merged image, arrow-metaphase plate, bar $-20 \mu \mathrm{m}$. (d-f) Double staining for F-actin with TRITC-phalloidin and for DNA with TO-PRO-3; (d) actin stained with TRITC-phalloidin; (e) chromosomes stained with DNA probe TO-PRO-3; (f) merged image, bar $-8 \mu \mathrm{m}$. (g-i) Double staining for actin by antibody against fragment of N-terminal domain of actin and for DNA by TO-PRO-3; (g) fluorescence of antibody-labeled actin; (h) DNA staining by TO-PRO-3; (i) merged image, bar-24 $\mu \mathrm{m}$. (j-k) Antibody against fragment of Nterminal domain of actin recognizes microfilaments near metaphase plate; (j) fluorescent staining with antibody, arrows-actin filaments; (k) double staining for actin by antibody and for DNA by TO-PRO-3, arrow-metaphase plate, bar $-8 \mu \mathrm{m}$. (m-n) After latrunculin B treatment antibody against N-terminus cannot detect actin filaments near chromosomes; (m) fluorescent staining with antibody; (n) double staining for actin by antibody and for DNA by TO-PRO-3, bar $-10 \mu \mathrm{m}$. (l and o) Disruption of cortical microfilament layer in oocytes after latrunculin B treatment; (l) F-actin stained with TRITC- phalloidin, bar $-24 \mu \mathrm{m}$; (o) actin fluorescence after labeling by antibody against fragment of $\mathrm{N}$-terminal domain, bar $-24 \mu \mathrm{m}$. 


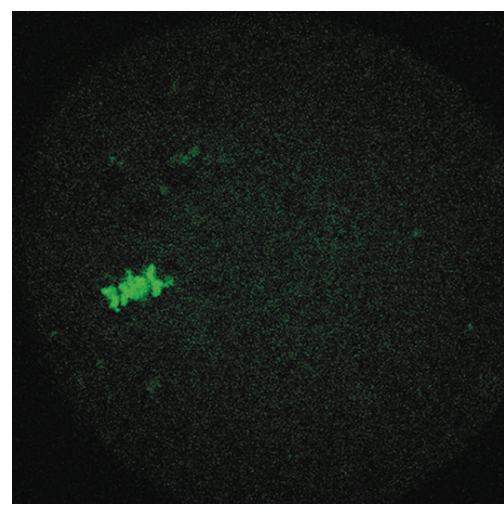

(a)

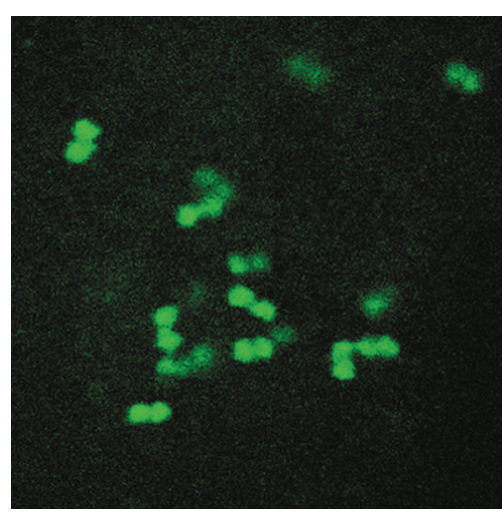

(d)

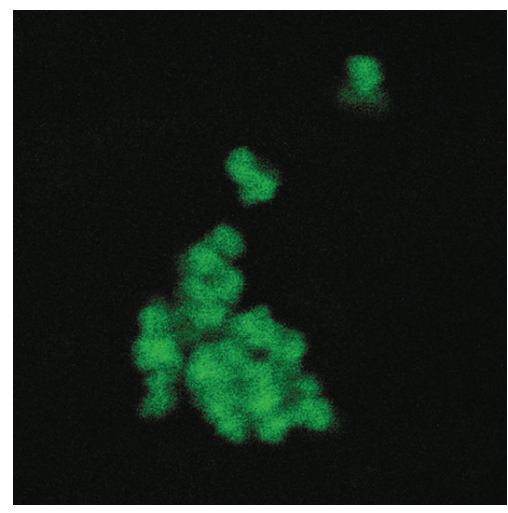

(g)

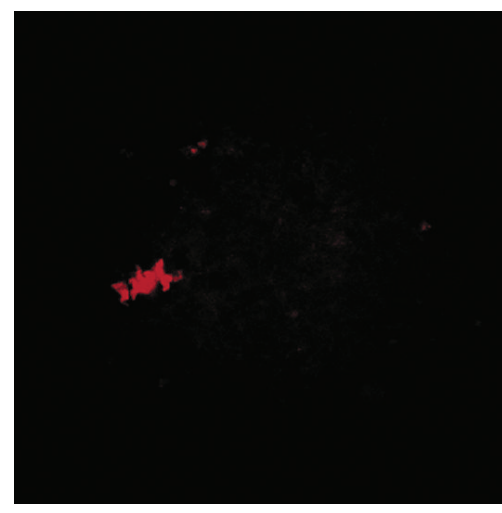

(b)

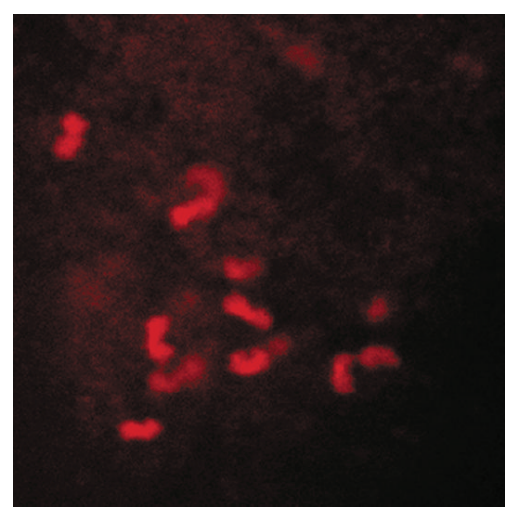

(e)

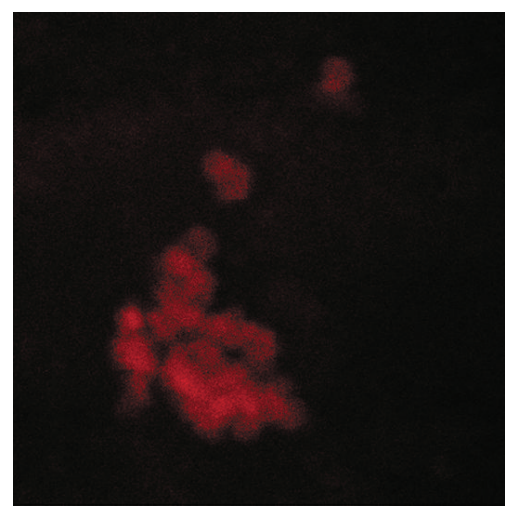

(h)

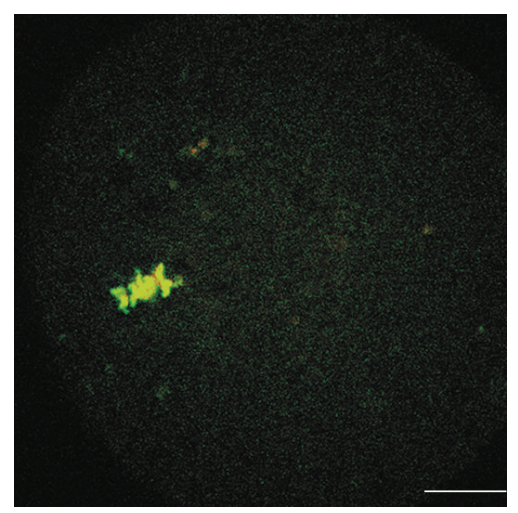

(c)

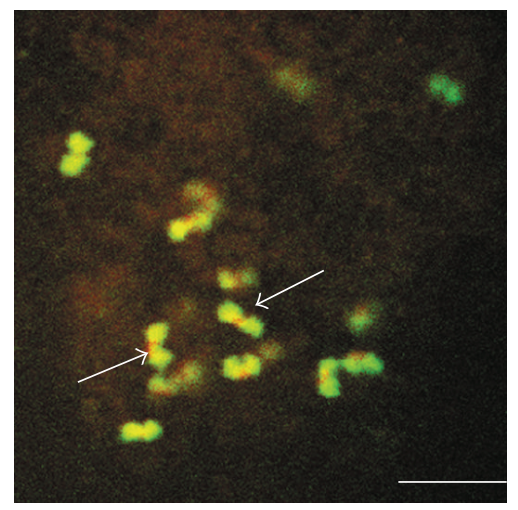

(f)

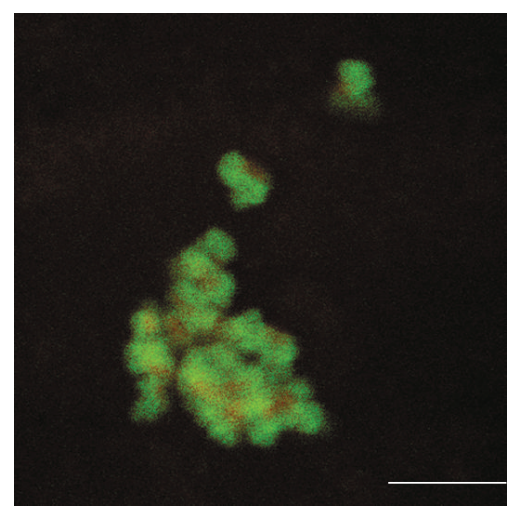

(i)

Figure 2: Colocalization of actin, labeled by antibody against fragment of C-terminal domain with metaphase chromosomes. (a-c) Colocalization of intact metaphase plate with actin; (a) fluorescent staining with antibody; (b) DNA staining by TO-PRO-3; (c) merged image, bar $-20 \mu \mathrm{m}$. (d-f) Colocalization of single chromosomes in destructed metaphase plate of squashed oocytes with actin: (d) actin staining with antibody; (e) DNA staining by TO-PRO-3; (f) merged image; arrow-centromere region where colocalization is absent, bar -8 $\mu \mathrm{m}$. (g-i) After latrunculin B treatment, the colocalization of actin with chromosomes was preserved; (g) fluorescent staining with antibody; (h) DNA staining by TO-PRO-3; (i) merged image, bar $-10 \mu \mathrm{m}$.

demonstrated a strong disassembly of the cortical microfilament layer (Figures 1(l) and 1(o)). Actin filaments that surround metaphase plate in intact oocytes (Figure 1(j)) were not observed in the cells exposed to latrunculin B (Figure 1(m)), and fluorescence of antibody in the cytoplasm around chromosomes was less bright than in untreated embryos (Figure $1(\mathrm{~m})$ ). In the same time, there were no changes in the distribution of actin detected by antibody to C-terminal domain in the oocytes after latrunculin B treatment, and actin-chromatin colocalization was preserved (Figure 2(i)).

Our observations of intact and latrunculin B-treated oocytes reliably demonstrated the presence of actin filaments close to metaphase plate of meiosis II and actin colocalization with metaphase chromosomes in mouse oocytes. Sensitivity of the filaments in spindle-like structure near chromosomes 
to the latrunculin B influence confirms their F-actin origin, but they may stay invisible for TRITC-phalloidin due to actin binding proteins connected with the microfilaments near phalloidin-binding sites.

As has been reported in several publications, actin polymerization is required during meiosis I for spindle assembly and motility [10-12]. We assume that in our study the antibody to N-terminal domain of actin has allowed demonstrating localization pattern of the microfilaments which provide meiosis II spindle migration and anchoring. In contrast to many mammalian species, mouse oocytes do not contain centrosomes and astral microtubules $[13,14]$ and spindle moving or stabile positioning require participation of actin microfilaments which directly interact with chromosomes $[15,16]$. Earlier spindle-like F-actin structure was described in anaphase I mouse oocytes [12], and now we observed actin filaments at metaphase II.

Confirmation of the tight association between actin and chromosomes was received from the study of isolated somatic human metaphase chromosomes [17]. Proteome analysis has demonstrated high affinity of actin for chromosomes, and immunostaining with monoclonal antibody to $\beta$-actin has discovered actin colocalization with chromosomes and visualized peripheral and speckled pattern of actin localization on chromosomes. On the basis of accumulated data, the assumption of close mutual relations of actin with the condensed chromosomes and participation of named protein in maintenance of chromosomal structural organization has been come out.

In our experiments, actin-chromatin colocalization was observed when actin was detected by antibody to the Cterminal domain, and this result is well correlated with the quoted above results of immunofluorescent analysis [17].

Demonstrated preservation of actin-chromatin colocalization after latrunculin-induced $\mathrm{F}$-actin depolymerization may be interpreted as evidence of the structural connection between actin and condensed chromatin.

At the same time it is still unclear which functional form of actin is colocalized with chromosomes if fluorescent markers to G- and F-actin do not show any signs of this. Earlier, studying actin localization in interphase nuclei of two-cell mouse embryos, we have found out that after embryos treatment by antibody to the $\mathrm{C}$-terminal domain, nuclear labeling was much brighter than cytoplasm fluorescence, and this signaled about predominant nuclear localization of antibodydetected actin [18]. Previously several authors described antibodies predominantly recognizing nuclear actin $[19,20]$, and we suppose that antibody to the C-terminal domain belongs to this type of antibodies.

Working with two-cell embryos we applied double staining: antibody to the C-terminal domain was used together with TRITC-phalloidin, or, as another variant, with Alexa Fluor 488-DNase I $[18,21]$. Results of these experiments demonstrated the visible discrepancy between the pattern of intranuclear actin fluorescence after staining by antibody and the distribution patterns of G-actin or F-actin ([21] and unpublished data), so we can assume that antibody to Cterminal domain is incapable for detecting monomeric and filamentous actin in mouse embryos.
In the nuclei of two-cell embryos, the actin detected by antibody to the C-terminal domain was observed mainly in the locations of highly condensed chromatin, in particular around nuclear precursor bodies, and it must be noted that during metaphase of the second embryo division antibodydetected actin was colocalized with chromosomes [21] in the same manner that we demonstrated here for meiosis II metaphase.

On the basis of the results received on oocytes and previously on embryos, we can come out with such assumption: some polymeric forms of nuclear actin exist in close and long-term association with condensed chromatin, and this association remains during cell division. This subpopulation of nuclear actin, possibly, may play a role in maintaining the structural organization of chromatin and in the process of chromatin condensation, as it was supposed earlier [22, 23].

\section{Acknowledgments}

This work was supported by the Russian Foundation for Basic Research (Project 10-04-00757) and The Program of Russian Academy of Sciences "Molecular and Cell Biology".

\section{References}

[1] B. T. Bettinger, D. M. Gilbert, and D. C. Amberg, "Actin up in the nucleus," Nature Reviews Molecular Cell Biology, vol. 5, no. 5, pp. 410-415, 2004.

[2] W. Hofmann, "Cell and molecular biology of nuclear actin," International Review of Cell and Molecular Biology, vol. 273, pp. 219-263, 2009.

[3] N. Visa, "Nuclear functions of actin," Cold Spring Harbor Perspectives in Biology, vol. 2, pp. 1-13, 2010.

[4] F. Miralles and N. Visa, "Actin in transcription and transcription regulation," Current Opinion in Cell Biology, vol. 18, no. 3, pp. 261-266, 2006.

[5] I. A. Olave, S. L. Reck-Peterson, and G. R. Crabtree, "Nuclear actin and actin-related proteins in chromatin remodeling," Annual Review of Biochemistry, vol. 71, pp. 755-781, 2002.

[6] M. T. Bohnsack, T. Stüven, C. Kuhn, V. C. Cordes, and D. Görlich, "A selective block of nuclear actin export stabilizes the giant nuclei of Xenopus oocytes," Nature Cell Biology, vol. 8, no. 3, pp. 257-263, 2006.

[7] K. Funaki, T. Katsumoto, and A. Iino, "Immunocytochemical localization of actin in the nucleolus of rat oocytes," Biology of the Cell, vol. 84, no. 3, pp. 139-146, 1995.

[8] E. Nguyen, D. Besombes, and P. Debey, "Immunofluorescent localization of actin in relation to transcription sites in mouse pronuclei," Molecular Reproduction and Development, vol. 50, no. 3, pp. 263-272, 1998.

[9] I. Spector, N. R. Shochet, Y. Kashman, and A. Groweiss, "Latrunculins: novel marine toxins that disrupt microfilament organization in cultured cells," Science, vol. 219, no. 4584, pp. 493-495, 1983.

[10] P. Lénárt, C. P. Bacher, N. Daigle et al., "A contractile nuclear actin network drives chromosome congression in oocytes," Nature, vol. 436, no. 7052, pp. 812-818, 2005.

[11] Q. Y. Sun and H. Schatten, "Regulation of dynamic events by microfilaments during oocyte maturation and fertilization," Reproduction, vol. 131, no. 2, pp. 193-205, 2006. 
[12] J. Azoury, K. W. Lee, V. Georget, P. Rassinier, B. Leader, and M. H. Verlhac, "Spindle positioning in mouse oocytes relies on a dynamic meshwork of actin filaments," Current Biology, vol. 18, no. 19, pp. 1514-1519, 2008.

[13] F. J. Longo and D. Y. Chen, "Development of cortical polarity in mouse eggs: involvement of the meiotic apparatus," Developmental Biology, vol. 107, no. 2, pp. 382-394, 1985.

[14] B. Maro, M. H. Johnson, M. Webb, and G. Flash, "Mechanism of polar body formation in the mouse oocyte: an interaction between the chromosomes the cytockeleton and the plasma membrane," Journal of Embryology and Experimental Morphology, vol. 92, pp. 11-32, 1986.

[15] J. van Blerkom and H. Bell, "Regulation of development in the fully grown mouse oocyte: chromosome-mediated temporal and spatial differentiation of the cytoplasm and plasma membrane," Journal of Embryology and Experimental Morphology, vol. 93, pp. 213-238, 1986.

[16] B. Maro and M. H. Verlhac, "Polar body formation: new rules for asymmetric divisions," Nature Cell Biology, vol. 4, no. 12, pp. E281-E283, 2002.

[17] S. Uchiyama, S. Kobayashi, H. Takata et al., "Proteome analysis of human metaphase chromosomes," Journal of Biological Chemistry, vol. 280, no. 17, pp. 16994-17004, 2005.

[18] N. A. Bogolyubova and I. O. Bogolyubova, "Actin localization in the nuclei of two-cell mouse embryos," Tsitologiya, vol. 51, no. 8 , pp. 663-669, 2009.

[19] S. M. Gonsior, S. Platz, S. Buchmeier et al., "Conformation difference between nuclear and cytoplasmic actin as detected by a monoclonal antibody," Journal of Cell Science, vol. 112, part 6, pp. 797-809, 1999.

[20] K. Milankov and U. De Boni, "Cytochemical localization of actin and myosin aggregates in interphase nuclei in situ," Experimental Cell Research, vol. 209, no. 2, pp. 189-199, 1993.

[21] N. A. Bogolyubova and I. O. Bogolyubova, "Early mouse embryos as a new model for studying of a functional role of the nuclear actin," Tsitologiya, vol. 52, no. 8, pp. 645-646, 2010.

[22] L. Goldstein, C. Ko, and J. Errick, "Nuclear actin: an apparent association with condensed chromatin," Cell Biology International Reports, vol. 1, no. 6, pp. 511-515, 1977.

[23] D. Rungger, E. Rungger-Brandle, C. Chaponnier, and G. Gabbiani, "Intranuclear injection of anti-actin antibodies into Xenopus oocytes blocks chromosome condensation," Nature, vol. 282 , no. 5736 , pp. 320-321, 1979. 

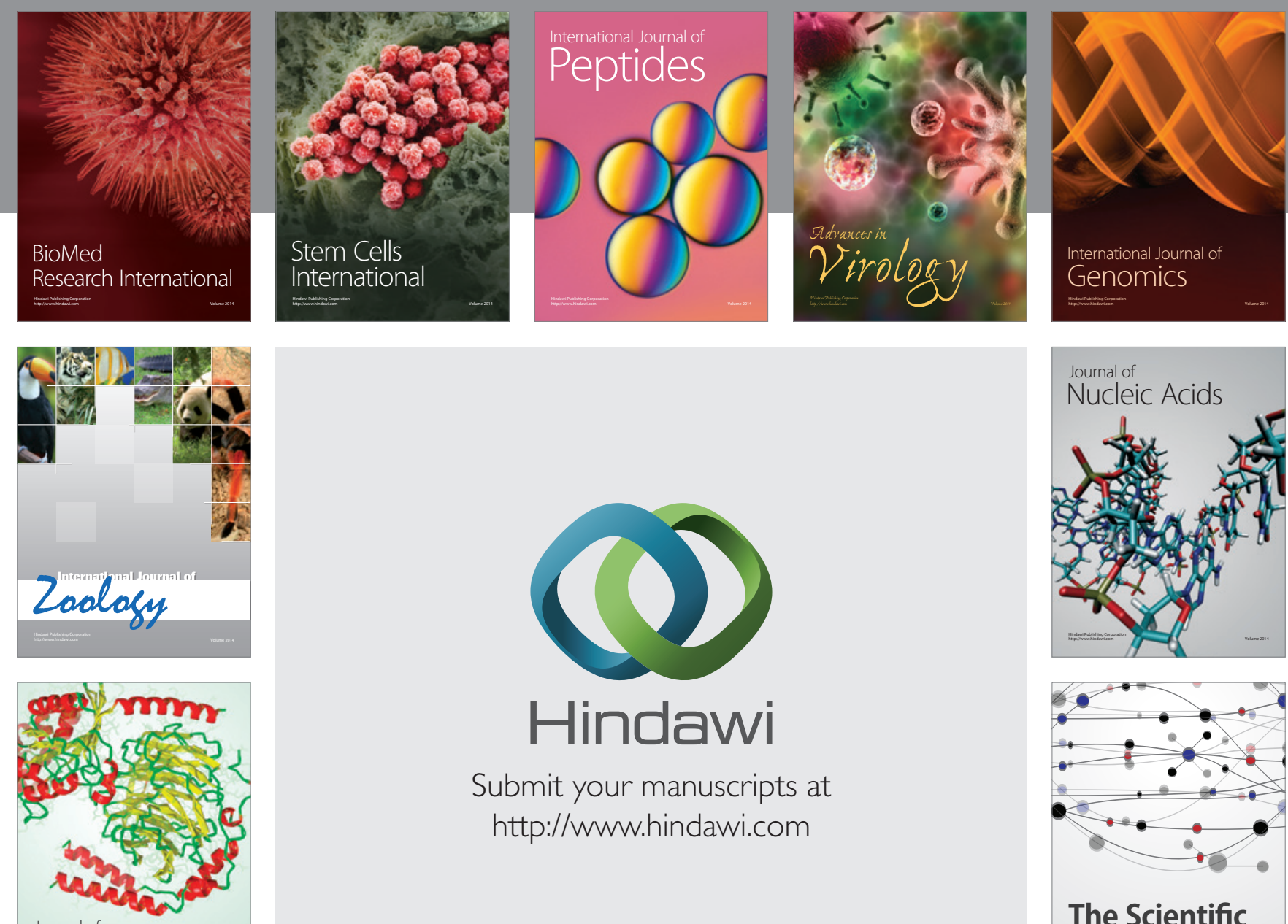

Submit your manuscripts at

http://www.hindawi.com

Journal of
Signal Transduction
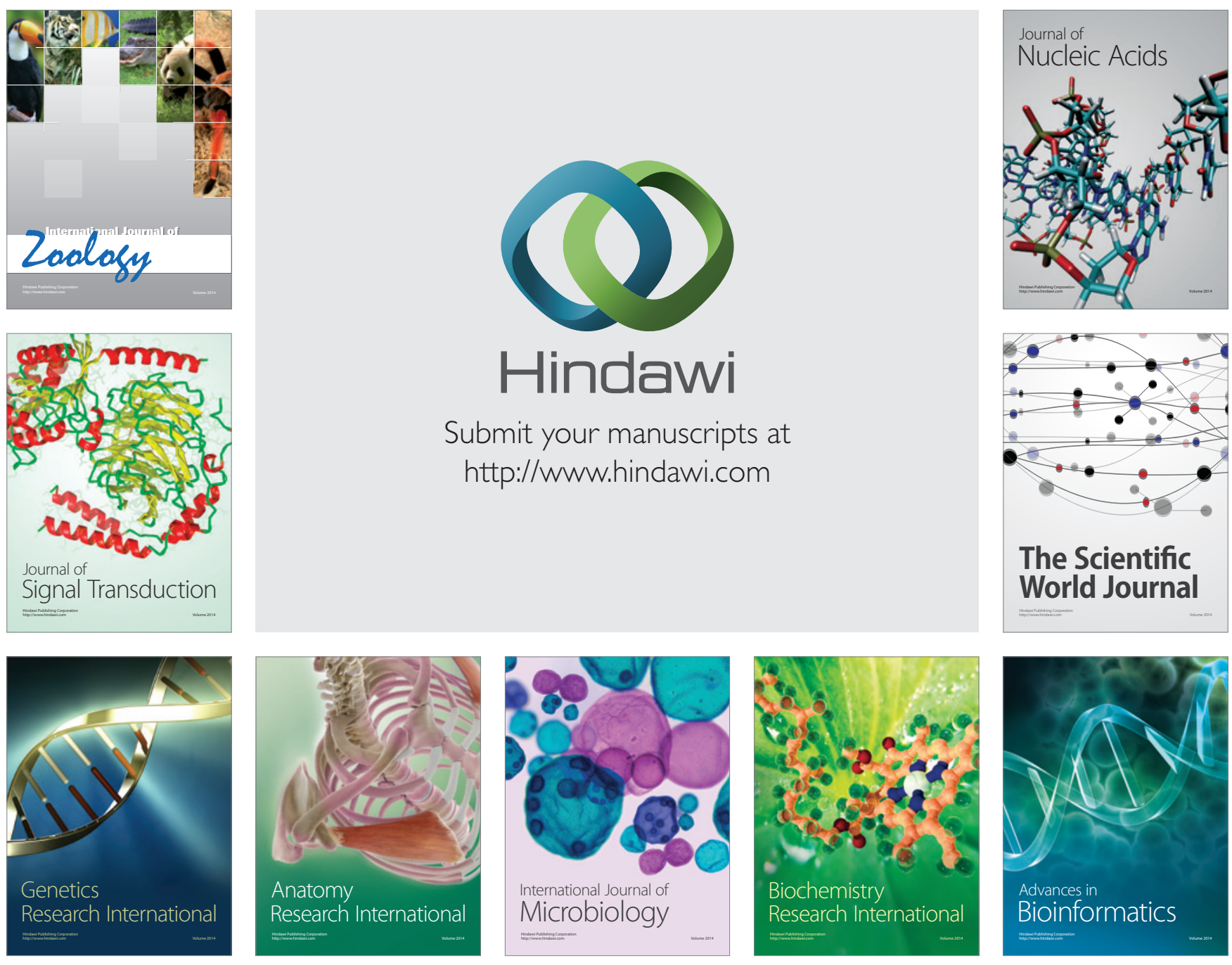

The Scientific World Journal
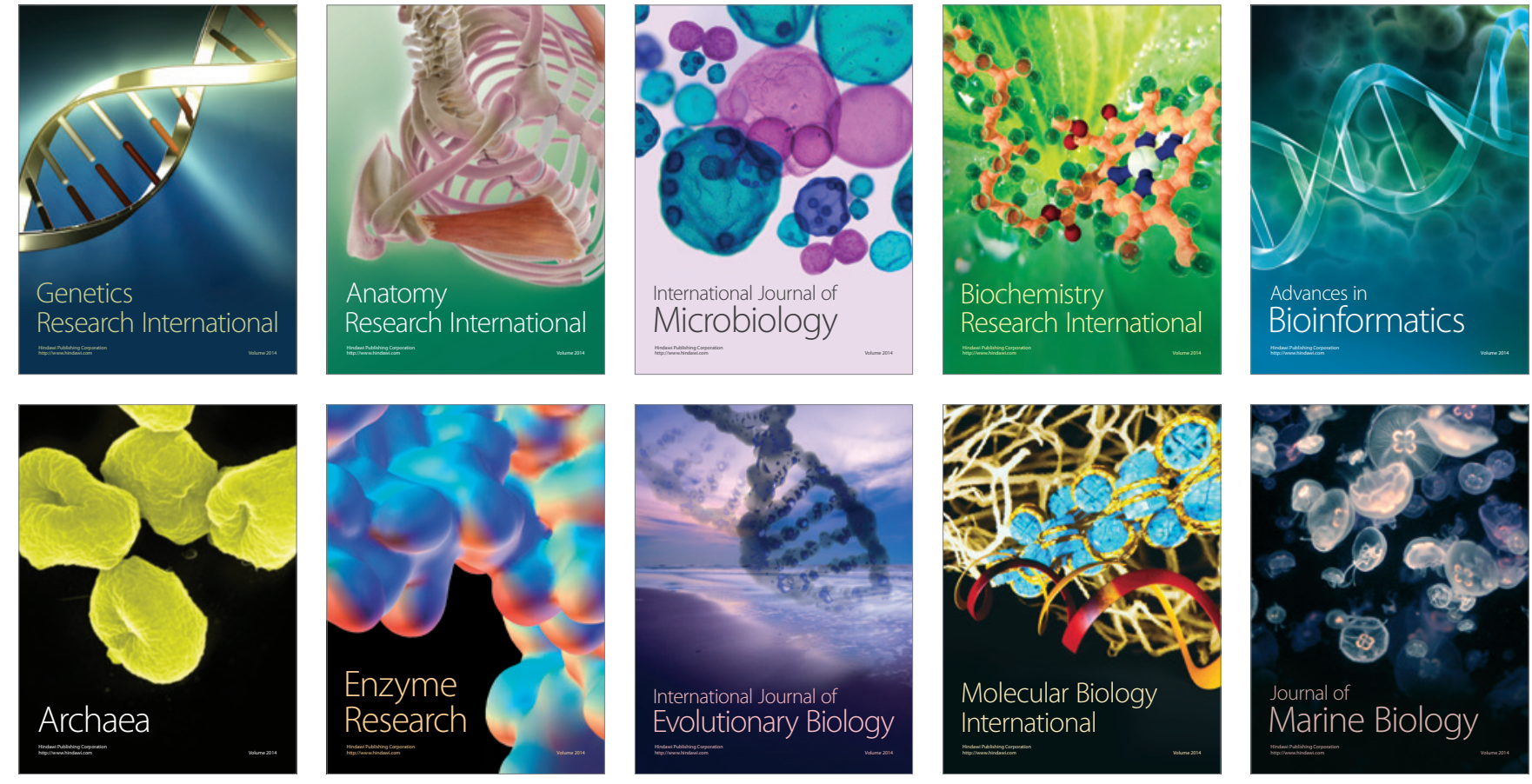